\title{
Towards Developing a Method for Identifying Static Compression Levels of Seamless Sports Bras using 3D Body Scanning
}

\author{
Adriana C. GOREA*, Fatma BAYTAR \\ lowa State University, Ames (IA), USA \\ DOI: $10.15221 / 16.022 \quad$ http://dx.doi.org/10.15221/16.022
}

\begin{abstract}
Sports bras are functional garments designed to minimize breast movements, while providing support, during physical activity. Understanding the factors influencing breast support is necessary for future development of better functioning sports bras to prevent wearers from soreness and pain. Compression was found to be the most effective factor in controlling the breast support. Moreover, respiratory state was reported to be significantly affecting bra size and comfort. Therefore, our pilot study explored how females' breast area measurements change when wearing seamless sports bras of different support levels. Two medium-size seamless sports bras with low and medium compression (i.e. breast support) levels were tested with and without removable pads. A $[T C]^{2} \mathrm{NX}-16$ three-dimensional (3D) body scanner was used to scan three females, with three different cup sizes, with and without wearing bras. Additionally, for each condition participants were scanned at fully inhaled and relaxed (neutral) positions. In order to identify compression rate changes among various conditions, twenty-one slice measurements taken at 0.25 inches intervals between under bust and armpit levels were collected and compared. The compression rates for each slice level were calculated by subtracting the slice circumference from the no bra circumference, and dividing the result by the no bra measurement. Our preliminary findings showed that the highest compression rates were achieved without pads, in both relaxed $(5.06 \%)$ and inhale conditions $(4.73 \%)$, at levels below bust height. Cumulative compression rates over all levels also showed higher compression rates for the bras without pads. Visual analyses revealed smoother contour lines and a more uniform distribution of compression all around the body for the medium support bra as compared to the low support bra for both with and without pads conditions.
\end{abstract}

Keywords: compression, 3D body scanning, sports bras

\section{Introduction}

The compression sportswear market is predicted to grow significantly in the next decade. Sports bras are functional garments specially designed to reduce repetitive movements of breasts during physical exercise. Continuous and repetitive movements can result in soreness, pain and irreversible tissue sagging [1]. Therefore, every woman, disregard of what size she is, should wear a supportive sports bra while running or exercising. However, a scientific and thorough understanding of the factors influencing breast support at rest as well as while exercising is necessary for future development of better functioning sports bras. This present study aims to investigate the use of three-dimensional (3D) body scanning technology as a tool to generate insightful data about static compression rate changes while wearing seamless sports bras.

\section{Literature Review}

The geometrical complexity of anatomical structure of women breasts makes designing bras with effective breast support, and creating accurate bra sizing very difficult. There is an abundant literature focusing on consumer satisfaction with current sports bras offerings [2-5]. Shoulder strap slippage, strap- cutting into the shoulder and band tightness around the chest area are the most important factors of bra discomfort [2]. An investigation regarding the comfort of sport bras found that bras offered the most support were the most uncomfortable ones at the same time [5]. Some studies specifically evaluated the performance of sports bras during physical exercises [5-7], and developed mechanical and bio-mechanical models to examine breast displacement in motion [8-10]. Existing literature revealed that bras act as external support mechanisms for the breasts, however, the relationship between breast mass and the bra size is very complex and yet to be determined. This is especially true for women with large breast sizes [8-10]. Although manufacturers specifically focus on sport kinematics when designing sports bras; bra materials, fabrics and specific manufacturing and construction processes also affect their expected performance. For example, an added layer of the cup

\footnotetext{
*agorea@iastate.edu; +1 302690 1842; www.iastate.edu
} 
pad slightly reduces the movement of the breasts during exercise [10], or fabric stretch influences the efficiency of a sports bra [8]. A recent study investigating sports bras identified the following common characteristics among different bra designs as factors influencing the support performance of the bra: compression type, high neckline, cross back, no center gore, no wire, no pad and a non-adjustable wide strap [13].

Axial loading that produces or squeezing or crushing is called compression. Previous studies classified clothing compression, or pressure, as static and dynamic [14]. Static pressure was imposed on one local area of the body when standing still. By contrast, dynamic pressure was exerted on a local body area during constant motion. Seamless knitting technology has been the primary technology used for manufacturing compression sport bras. Eliminating seams, especially cup and side seams, provides not only comfort, but also performance support by achieving uniform compression levels around the body $[14,15,22,23]$. This technology also brings new stitching patterns and knitting structures to create 3D forms, that furthermore proved to affect the compression levels [16]. Compression garments for sportswear have become widely available providing increased comfort, fit and muscle support [12]. Many athletes consider compression of muscles to support and improve muscle alignment [11].

Determining bra sizing methodology has been another challenge within the compression bra industry [11]. Most brands develop a sizing system based on the seamless knitting technology that they have available. The range of diameters of the circular knitting machines used for compression sports bras is limited. Therefore, it became a popular practice to offer standard sizes labeled Extra Small, Small, Medium, Large and Extra Large, without adding a cup size limitation as with the regular bra sizing systems [25]. Since 1935, the bra sizing system has been based on only two measurements: bust girth and under bust girth. The difference between these two measurements is designated as a cup size label. However, in the seamless bras category, the cup size designation cannot be accurate since the geometry of the cup of the breast is not considered in the design of the seamless bras [25]. The respiratory state has also been found to affect bra size determinations [26,27]. The results showed that, the difference between under bust band circumference during inhale versus exhale ranged from 0 to 6 inches, with a mean standard deviation of 1.9 inches. In both respiratory states, cup size was found to be underestimated when compared with the self-reported cup size of each participant, and under bust band size was found to be overestimated [26]. The same study found that, given the variations in women postures and breast shapes, self-reported bra sizes are often incorrect since visual estimation of full breast level during various respiratory states is inaccurate. The proposed solution was to take measurements in various respiratory conditions and average them to identify optimum bra size [26].

Evaluating static compression of seamless knitted sports bras is challenged by the lack of accuracy of the measurement devices on the market, and the convenience of their operation [14]. The use of 3D body scanning technology has been successfully used in conjunction with determining precise body contours, such as the complicated geometry of the breast area [3]. However, no research has been found to use 3D body scanning as means of evaluating and comparing compression levels in various areas of a seamless sports bra. Therefore, the purpose of this pilot study was to develop a methodology to investigate how breast area changes while wearing various seamless compression sports bras in different respiration states.

\section{Methods}

After obtaining an Institutional Review Board approval for testing, three subjects were invited to the scan lab. The subjects were voluntary female students at a Midwestern university, ages varied in between 25-40 years old, with wearing size $\mathrm{M}$ sports bras. No other size conditions were considered in the selection of the subjects, as they do not affect compression levels at the bust area. Two of the subjects (Subjects 1 and 2) were Caucasian Americans, with lower exercise levels (2 out of 10); while the third subject (Subject 3) was an Asian American and had the highest exercise level (10 out of 10). Subjects 1 and 2 had kids whereas Subject 3 did not have any kids.

A Textile/Clothing Technology Corporation [TC $]^{2}$ NX-16 3D body scanner was used to collect data for this pilot study. In addition to the basic bust area girth measurements such as under bust and bust, scan slices provided by the scanner's software enabled the visualization of the body in a much deeper manner than surface analysis. Scan slices are often used to visually represent changing size and circumferences [29].

Two compression seamless sport bras with low breast support and moderate breast support were tested. They were chosen in manufacturer's size $\mathrm{M}$, fitting a wide range of wearers with various cup sizes. The style of both bras were similar, with skinny straps, inserted removable pads and racer 
back criss-crossings. It is a common practice among the main sports bras manufacturers to provide seamless sports bras with inserted pockets for removable pads. As the literature review highlighted, the addition of bra pads has been questionable in adding extra support during physical exercise. Their use is mostly for adding shape to the otherwise flat look achieved after compressing the bust area [7]. The removable feature of the pads is a manufacturing solution for inserting the foam pads after the seamless bra has been knitted. Ideally, the added support offered by the pad addition should be engineered into the knitting pattern and yarn design. Sports bras are designed to provide three levels of support, i.e. low, medium and high, based on the activity types. Low impact support may be sufficient for walking, yoga, and strength training; whereas medium support may be needed for hiking, road cycling, skiing; and high support may work better for high-impact activities such as running, aerobics, mountain hiking.

The first seamless sports bra tested was designed for low impact, made of $71 \%$ : Nylon, $24 \%$ : Polyester, $5 \%$ : Spandex. Other features advertised by the manufacturer are "a compressive fit, adjustable straps and wicking fabric that dries fast". The second bra tested was characterized by the manufacturer as "medium-impact racerback bra, removable cups add shape and support", made of $55 \%$ Nylon / 36\% Polyester / 9\% Spandex (Figure 1).

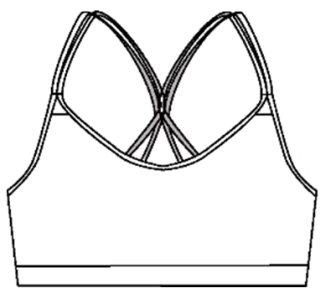

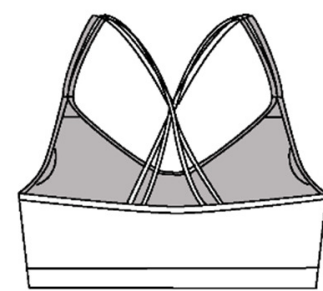

(a)

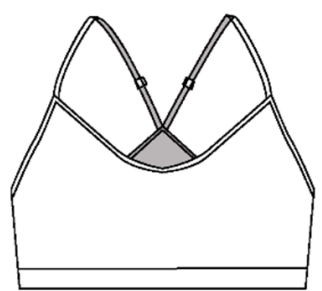

(b)

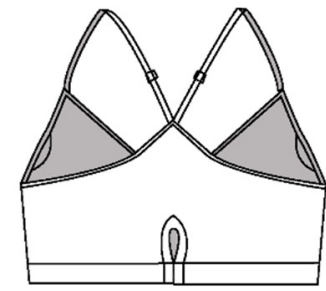

Fig. 1 Tested sports bras samples: (a) low support style, and (b) medium support style

According to the previous research, respiratory state significantly affects bra size and comfort and it should be considered when evaluating breast measurements [27]. Therefore, data was collected during 5 random bra conditions: (1) no bra, (2) the low-support bra without the pads (3) the low-support bra with the pads, (4) medium-support bra without the pads and (5) medium support bra with the pads [7]. Two scans were taken for each of the above mentioned conditions: end voluntary inspiration (subjects held breath for the 15 seconds duration of the scan) and relaxed voluntary expiration (regular breathing with minimal accessory muscle effort). The wearer was in an upright position, with both arms placed on the scanner handles. For the bottom of the body, a pair of scanning knee length pants were given to subjects to wear for all conditions. Compression bras are made from seamless knits and compress the breasts against the chest wall to restrict movement. They do not have cups built into the design and tend to work best for low- to medium-impact activities.

For each subject, our aim was to extract and compare the cross sectional maximum girth of different areas along the surface of the sports bras, in the ten different bra and breathing situations. Moreover, we looked at the cross sectional areas of bust areas in all scan conditions. The starting slice was determined by the lowest level (relative to the floor) of the "Under Bust Full" slice measurement provided by the scanner software. Slice contours taken at 1 inch intervals from under bust to armpit level were nested and compared to visually examine shape variations for mapping compression characteristics of the two different levels of bra support. Twenty-one slice measurements taken at 0.25 inches intervals between under bust and armpit levels were collected for each scan condition. As subjects' overall height varied, the bottom level used to create slices were adjusted for each subject differently based on the scanner's under bust height measurement result. A rate of change, similar to a growth rate, is a rate that describes how one quantity changes in relation to another quantity. In our study, the rate of change in slice circumferences for each height level were calculated by subtracting the slice circumference from the no bra circumference, and dividing the result by the no bra circumference measurement, for each subject, bra, pad condition and respiratory state, as shown in equation 1 , where $b$ indicates slice circumference in no bra condition, and $a$ indicates slice circumference in a particular experimental condition $(n)$.

$$
\text { Rate of change of slice circumference }{ }_{n}(\%)=\frac{b-a}{a} \times 100
$$

In this study, compression rate refers to the rate of change in slice circumference relative to the no bra condition. 


\section{Results and Conclusions}

Our pilot study findings showed that the highest compression rates were achieved in the without pads condition, in both relaxed (5.06\%) and inhale conditions (4.73\%), at levels below bust height. This is a plausible finding since functional apparel design literature suggests that added pads may absorb the compression forces, therefore the lack of pads may be the cause of higher compression on malleable bra tissue [10]. Considering the suggestions from previous studies, the slice measurements from relaxed and inhale conditions were averaged for all scans and subjects at each slice level [26]. Data collected beyond 4 inches above the under bust level were not considered, as the subjects' body position during the scan had their arms touching the body at the armpit level, overestimating the slice circumferences at that levels. For this reason, only 17 out of 21 collected measurements were included in the analysis of each scan. The compression rates for Subject 3, who had the largest full bust circumference (38.5 inches) among of all, were different from the compression rates observed from the other two participants. While the two participants with lower full bust circumference measurements (37.5 and 38 inches) had positive compression rates, the participant with the larger full bust measurement had a few negative rates. In other words, the smaller breasts got compressed more while for the larger breasts breast tissue gets relocated within a compression bra. This resulted in slice measurements while wearing compression bras to be bigger than the same measurements while wearing no bra. This type of data should not be neglected; therefore, negative compression rates were not eliminated from the calculations for each bra condition.

After averaging the data for each slice level, the highest compression rate was achieved by the low support bra without the pads (3.44\% at 0.75 inches above under bust level). The lowest average compression rate was achieved in the medium support with the pads condition (1.62\%) at just 0.25 inches above the under bust level. The box chart below (see Figure 2) shows that the distribution of average compression rates is within smaller interval values for the medium support bra as compared to the low support bra. For each condition, the ranges changed as follows: (1) $0.55 \%$ to $2.52 \%$ for the medium support bra no pads, (2) $-0.27 \%$ to $1.62 \%$ for the medium support bra with pads, (3) $-1.1 \%$ to $3.44 \%$ for the low support bra no pads, and (4) $0.78 \%$ to $2.82 \%$ for the low support bra with pads. The wider range of variability for average compression rates for the low support bra with no pads suggests that the bra material controls less breast tissue. The presence of pads seems to limit the range of compression rate for both medium support and low support bras. However, the median value of compression rate is the highest for the low support bra with no pads $(2.8 \%)$, showing a more effective compression among all three subjects.

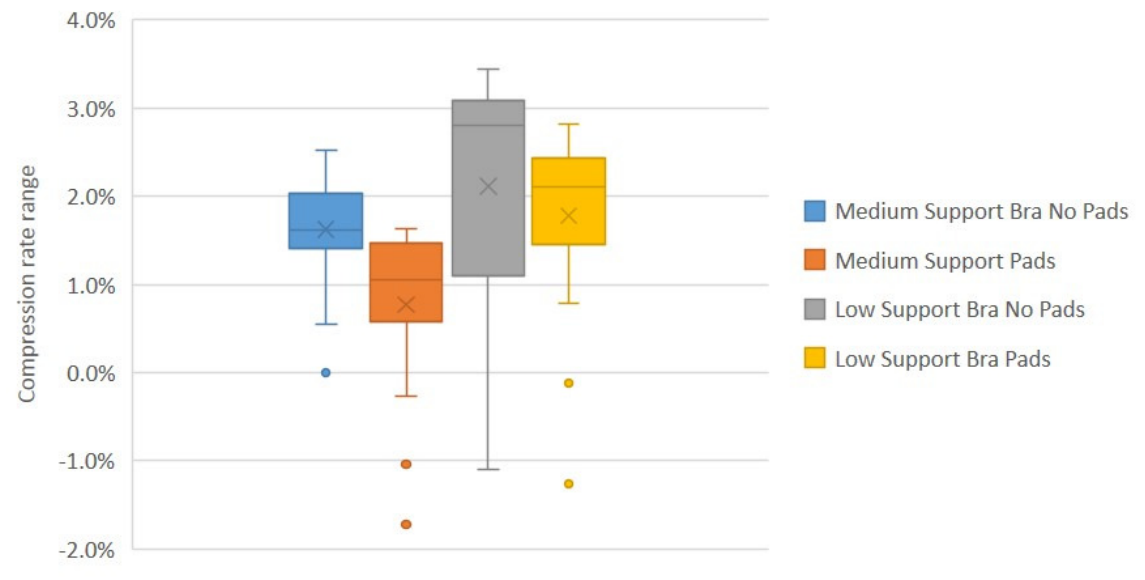

Fig. 2. Average compression rate distribution among the four scan conditions for all subjects.

Furthermore, totaling data for all slice heights then averaging, low support bra no pads was found to give the highest total compression rate of $2.11 \%$, versus $0.77 \%$ as the lowest total compression rate for the medium support bra with pads. The total results for the other two conditions, low support bra with pads was $1.49 \%$, while for the medium support bra no pads was $1.63 \%$. A line plot of the average compression rates for each bra showcasing the pads as opposed to no pads condition is shown below (see Figure 3). 


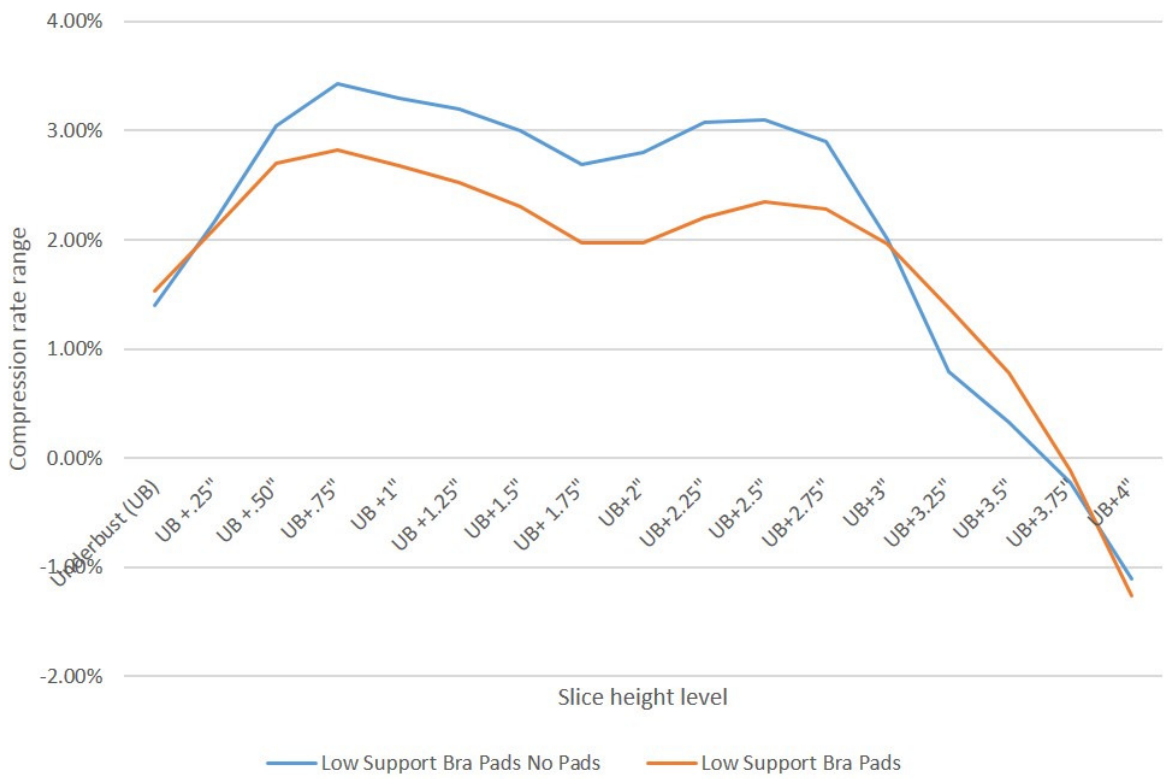

(a)

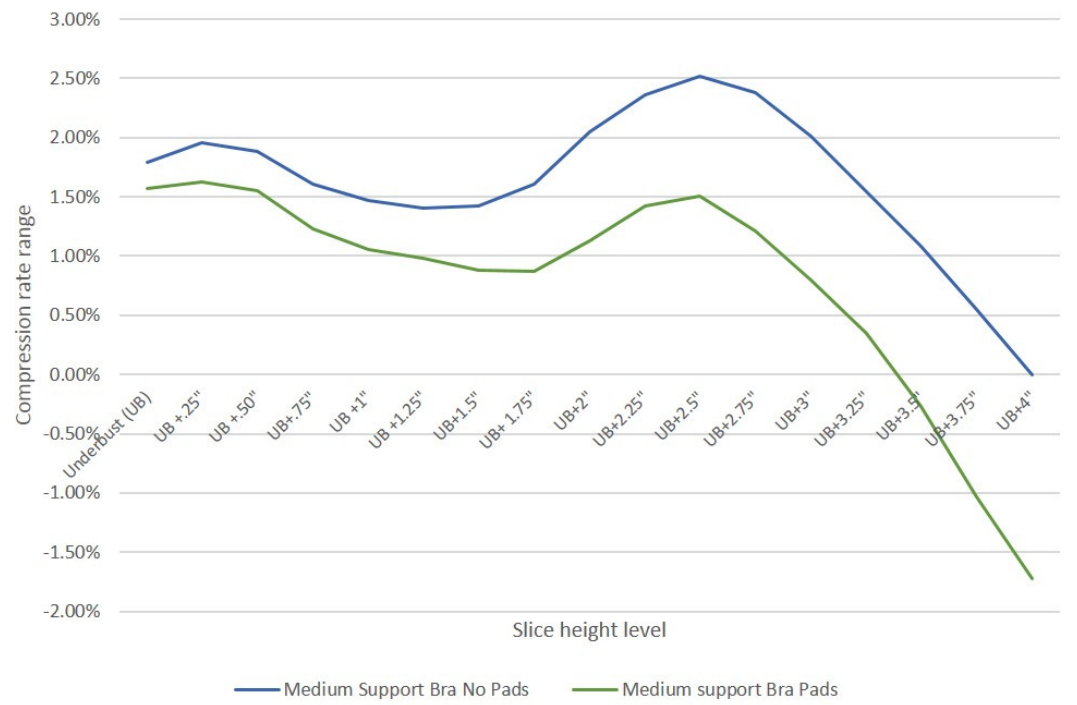

(b)

Fig. 3 Comparison of average compression rates of pads versus no pads conditions for (a) low support bra and (b) medium support bra.

The two lines of each plot show that the pads did not add to the compression, but rather lowered the compression rates. Also, the patterns show how the compression rates varied along the slice levels, achieving higher compression rates around full bust (under bust +2.5 inches) for the medium support bra, while for the low support bra the higher compression rates were around under bust (under bust +0.75 inches).

Visual analyses revealed smoother contour lines and a more uniform distribution of compression all around the body for the medium support bra as compared to the low support bra for both with and without pads conditions (Figure 4). This finding may also suggest that the medium support bra might be made from a relatively thicker fabric that is less reflective of all the skin folds. 


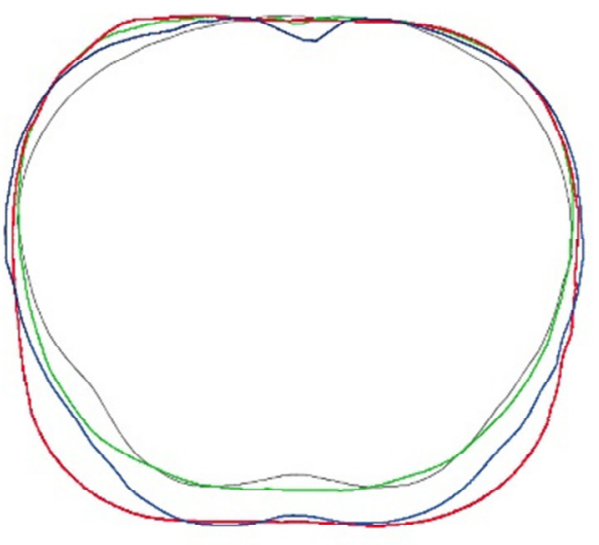

(a)

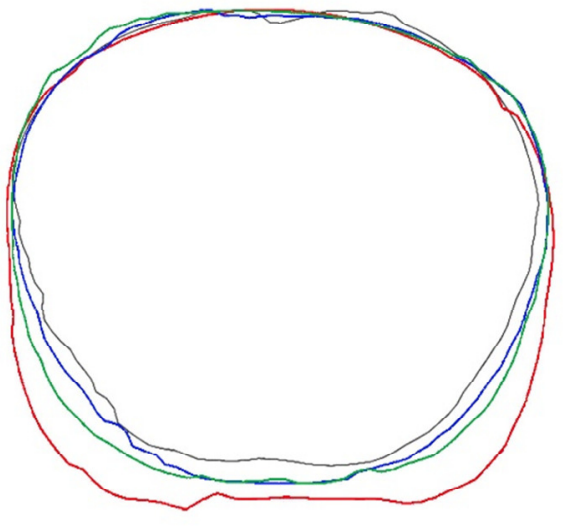

(b)

Fig. 4. Four consecutive slices between under bust and bust levels (with pads in inhale condition) showing (a) uniform compression distribution around the body for medium support bra, and (b) contour irregularities for low support bra. Data taken from Participant 1.

Cumulative compression rates over all levels showed higher compression rates for the bras without pads. These results confirm that different constructions of sports bras affect the way the breast area is distributed and supported during static phase, and the labeling of the support level does not necessarily reflect the actual compression benefit for the wearer. The inconsistent findings between compression rates of bras with pads and without pads raise the question of the utility of the extra layers in the bra, besides the smoothing of the body contour as shown in Figure 3 . The thickness of the pads ( 0.25 inches at the thickest part), while minimally adding to the circumference of the slices it might add more to the volume of the breast area. This could be further investigated as to establish the real benefits as related to breast support.

\section{Limitations}

In this pilot test, we explored the accuracy and type of data that can be derived from 3D body scanning while wearing compression sports bras. Although we had only three participants, we were able to see the capability of the 3D body scanner and refine our test procedures for our main study. In order to be able to see the compression bra-related changes, a further study should recruit participants with similar characteristics. Scanning results would vary for women in different ages, who had kids, went through cancer treatment, ethnicity, exercise level, and so forth. Moreover, the two bras that were tested had slightly different knitted stitch patterns. Literature showed that knitting patterns may affect wearing compression results [16]. Multiple factors involved in bra sizing and fitting as well as the variety of body shapes and exercise types add complexity and limitation to such study. Also, it might be likely that the subjects moved the bra pads and adjusted the under bust band to reach the desired comfort level without even realizing it. We did not have a control over this during our pilot tests.

It should be also mentioned that among several 3D body scanner types that are currently available, significant variance exists in how each one of them captures specific body measurements. Besides, there is no clear information about how the scanner identifies the under bust and full bust height. Further research should be done on evaluating static compression using 3D body scanning in various postures during physical exercise, aiming to eventually plot out a methodology for evaluating the dynamic pressure.

\section{References}

[1] R. Zheng et al., "Development of a New Chinese bra sizing system based on breast anthropometric measurements.", in International Journal of Industrial Ergonomics, Vol.37, No.8, 2007, pp. 697-705, http://dx.doi.org/10.1016/..ergon.2007.05.008

[2] K.A. Bowles et al., "Features of sports bras that deter their use by Australian women." in Journal of Science and Medicine in Sport 15, No. 3, 2012, pp. 195-200.

[3] C.M. Chen et al., "Physical characteristics related to bra fit." in Ergonomics, Vol. 53, No. 4, 2010, pp. 514-524. 
[4] B.R. Mason et al., "An analysis of movement and discomfort of the female breast during exercise and the effects of breast support in three cases." in Journal of Science and Medicine in Sport, Vol.2, No. 2, 1999, pp. 134-144.

[5] D.E. McGhee et al., "Optimising breast support in female patients through correct bra fit. A cross-sectional study." in Journal of Science and Medicine in Sport, Vol.13, No. 6, 2010, pp. 568-572.

[6] J. Zhou et al., "Evaluation of shock absorbing performance of sports bras." Journal of Fiber Bioengineering and Informatics, Vol. 2, No. 2, 2009, pp. 108-113.

[7] J.C. Scurr et al., "Supported and unsupported breast displacement in three dimensions across treadmill activity levels." in Journal of sports sciences, Vol. 29, No. 1, 2011, pp. 55-61.

[8] Y. Li et al., "A 3D biomechanical model for numerical simulation of dynamic mechanical interactions of bra and breast during wear." in Journal of the Textile Institute, Vol. 95, No.8, 2004, pp.59-79.

[9] D.E. McGhee et al., "Bra-breast forces generated in women with large breasts while standing and during treadmill running: Implications for sports bra design." in Applied ergonomics, Vol. 44, No. 1, 2013, pp. 112-118.

[10] M. Lu et al., "Mechanical analysis of breast-bra interaction for sports bra design." in Materials Today Communications, Vol. 6, 2016, pp. 28-36.

[11] P. Venkatraman, and D. J. Tyler. "Applications of Compression Sportswear." in Materials and Technology for Sportswear and Performance Apparel, 2015, pp. 171-203.

[12] J. Voyce et al., "Elastic textiles." in Textiles in sport. Cambridge: Woodhead Publishing Limited, 2005, pp. 204-30.

[13] J. Zhou et. al, "Identifying effective design features of commercial sports bras." in Textile Research Journal , 2012, http://tri.sagepub.com/content/early/2012/11/23/0040517512464289

[14] R. Zheng et al., "Pressure evaluation of 3D seamless knitted bras and conventional wired bras." in Fibers and Polymers, Vol.10, No. 1, 2009, pp. 124-131.

[15] S. Luo et al., "A novel approach to characterize dynamic pressure on lower limb wearing compression cycling shorts." in The Journal of The Textile Institute, Vol. 107, No. 8, 2016, pp. 1004-1013.

[16] S.K.Tiwari et al., "A pilot study: Evaluating the influence of knitting patterns and densities on fabric properties for sports applications." Procedia Engineering , Vol. 60, 2013, pp. 373-377.

[17] J. Al Khaburi et al., "Measurement of interface pressure applied by medical compression bandages." in 2011 IEEE International Conference on Mechatronics and Automation, IEEE, 2011, pp. 289-294.

[18] N. Brophy-Williams et al., "Evaluating the Kikuhime pressure monitor for use with sports compression clothing." in Sports Engineering, Vol.17, No. 1, 2014, pp. 55-60.

[19] F. Wang et al., "Flexible pressure sensors for smart protective clothing against impact loading." Smart Materials and Structures, Vol. 23, No. 1, 2013, 015001.

[20] M. Sung et al., "Suggestion for optimal location of textile-based ECG electrodes on an elastic shirts considering clothing pressure of the shirt." in 2008 12th IEEE International Symposium on Wearable Computers, IEEE, 2008, pp. 121-122.

[21] H. Morooka et al., "Development of a device using cylinder method for measuring clothing pressure of elastic socks and pantyhose." In SEN-I GAKKAISHI, Vol. 63, No. 5, 2007, pp. 130-137.

[22] R. Mahbub et al., "Design of knitted three-dimensional seamless female body armour vests." in International Journal of Fashion Design, Technology and Education, Vol. 7, No. 3, 2014, pp. 198-207.

[23] C. Radvan, "Inclusively designed womenswear through industrial seamless knitting technology." in Fashion Practice, Vol. 5, No. 1, 2013, pp. 33-58.

[24] Y. Lee and K. Hong, "Development of indirect method for clothing pressure measurement using three-dimensional imaging." in Textile Research Journal, Vol. 83, No. 15, 2013, pp. 1594-1605.

[25] R. Zheng et al., "Development of a new Chinese bra sizing system based on breast anthropometric measurements." in International Journal of Industrial Ergonomics, Vol. 37, No. 8, 2007, pp. 697-705.

[26] D.E. McGhee and J. R. Steele. "How do respiratory state and measurement method affect bra size calculations?" in British journal of sports medicine, Vol. 40, No. 12, 2006, pp. 970-974.

[27] K.A. Bowles et al., "Do current sports brassiere designs impede respiratory function?" in Medicine and science in sports and exercise, Vol. 37, No. 9, 2005, pp. 1633-1640. 